\title{
Comportamiento de la Fibra de Celulosa Reciclada en el Proceso de Hidratación
}

\author{
José Turrado, Alma R. Saucedo, Juan Ramos y Maria L. Reynoso \\ Universidad de Guadalajara, Departamento de Madera, Celulosa y Papel Ing. Karl Augustin \\ Grellmann, Km 15.5 carretera Guadalajara-Nogales, 45000 Zapopan, Jalisco - México \\ (e-mail: jturrado@dmcyp.cucei.udg.mx)
}

\section{Resumen}

En este trabajo se estudia el proceso de hidratación en fibra modificada por el proceso de reciclado. Para ello se emplea pulpa kraft de primer ciclo (muestra A) y se compara con fibra kraft del ciclo $n$ (muestra B). Ambas fibras por separado se someten a proceso de hidratación. Posteriormente las muestras $A$ y $B$ se mezclan al $50 \%$, generando una mezcla fibrosa para la estructuración de una nueva hoja de papel. Como factores de respuesta se determina: resistencia a la tensión, elongación, absorción de energía de tensión y valor de retención de agua $\left(\mathrm{g}_{\text {agua }} / \mathrm{g}_{\mathrm{fibra}}\right)$. La hidratación se realiza con agua, hidróxido de sodio y dosificación de dos agentes de tensión superficial con carácter hidratante. En agua la mezcla de fibras alcanza su máxima capacidad de absorción $\left(1,2 \mathrm{~g}\right.$ agua $\left./ \mathrm{g}_{\mathrm{fibra}}\right)$ en $18 \mathrm{~h}$, en solución alcalina $\left(1,4 \mathrm{~g}\right.$ agua $\left./ \mathrm{g}_{\mathrm{fibra}}\right)$ en $15 \mathrm{~h}$ y con agentes de tensión superficial este máximo $\left(1,3 \mathrm{~g}_{\text {agua }} / \mathrm{g}_{\mathrm{fibra}}\right)$ se alcanza en $1 \mathrm{~h}$.

Palabras claves: hidratación, fibra reciclada, pulpa kraft, agentes de tensión superficial

\section{Behavior of Recycled Cellulose Fiber during the Hydration Process}

\begin{abstract}
In this work the phenomenon of hydration is studied in recycled fiber. As raw material kraft of the first cycle (sample $A$ ) is used and results compared with recycled kraft fiber of the $n$ cycle (sample $B$ ). Both fibers are separately treated under hydration conditions. Later, fibers A and B are mixed, $50 \%$ each, generating a fibrous mixture to manufacture a new paper sheet. As response factors the following were evaluated: tensile resistance, elongation, tensile absorption energy and water retention value $\left(\mathrm{g}_{\text {water }} / \mathrm{g}_{\text {fiber }}\right)$. The hydration is carried out with: water, sodium hydroxide and two surface active agents with moisture activity. In water the mixture of fibbers reaches its maximum capacity of water absorption $\left(1,2 \mathrm{~g}_{\text {water }} / \mathrm{g}_{\text {fiber }}\right)$ in $18 \mathrm{~h}$, in alkaline solution $\left(1,4 \mathrm{~g}\right.$ water $\left./ \mathrm{g}_{\text {fiber }}\right)$ and in surface active agent $\left(1,3 \mathrm{~g}_{\text {water }} / \mathrm{g}_{\text {fiber }}\right)$ in $1 \mathrm{~h}$.
\end{abstract}

Keywords: hydration, recycled fiber, kraft pulp, surface active agent 


\section{INTRODUCCIÓN}

A nivel mundial, la comercialización de fibra celulósica se hace en su forma casi seca. Por lo que la producción de papel en muchos casos se efectúa a partir de fibra que ha sido sometida a un proceso de secado. Aunado al proceso de comercialización de fibra obtenida a partir de papel post consumo.

En el papel post consumo, la fibra sufre diversas modificaciones que están directamente relacionadas con su estructura. En la pared celular externa se produce un endurecimiento que impide el completo humedecimiento de la fibra, es decir, se pierde la capacidad de absorción de agua e hinchamiento de la fibra y por lo tanto la flexibilidad para la estructuración del nuevo papel.

Los cambios que ocurren en la fibra durante el proceso de secado corresponden a un fenómeno investigado en fibra virgen por Jayme (Jayme, 1994), al que se denominó Hornificación. Este término se refiere a las alteraciones que sufren las capas externas de la fibra celulósica durante el proceso de secado en la fabricación de papel y durante su exposición al medio ambiente. Estos cambios inducen a diferencias en propiedades de resistencia del papel, en comparación con el papel que se ha estructurado a partir de pulpa que no se ha secado nunca, especialmente cuando se evalúan propiedades que están directamente relacionadas con la hidratación e hinchamiento de la fibra celulósica.

Si el fenómeno de Hornificación ha ocurrido, este fenómeno es irreversible de acuerdo a la definición original, sin embargo, los estudios de Fengel y Wegener (1984) y Minor (1992) indican que el endurecimiento de las fibras se puede reducir al utilizar métodos que rompan los enlaces "puentes de hidrógeno" o interferir con su formación, ya sea por una sustitución parcial de los grupos hidroxilos de la celulosa por grupos que no formen puentes de hidrógeno tanto con la fibra como consigo mismos; o bien secar la fibra en presencia de aditivos que proporcionen volumen a la pared celular y de esta forma proteger al gel ligno-hemicelulósico.

La organización típica de una fibra vegetal de acuerdo a Stone y Scallan (1965), se muestra en la Fig. 1 y se observa que entre las fibras existe una región denominada lámela media "Sustancia intercelular", la cual esta constituida principalmente por lignina y sustancias pépticas. La pared primaria $\mathrm{P}$ contiene una red organizada en forma suelta y aleatoria de microfibrillas de celulosa embebidas en una matriz de hemicelulosas parcialmente orientadas. Inmediatamente bajo la pared primaria está la pared secundaria, la que constituye casi la totalidad de la pared celular. La pared secundaria está dividida en tres capas denominadas $S_{1}, S_{2}$ y $S_{3}$. La capa exterior de la pared secundaria $S_{1}$ presenta un patrón entrecruzado de microfibrillas. La capa $S_{2}$ ocupa la mayor parte del volumen de la pared celular, en esta parte las microfibrillas están orientadas en forma casi paralela al eje de la fibra. En la capa delgada $S_{3}$ las microfibrillas forman una hélice plana en dirección transversal.

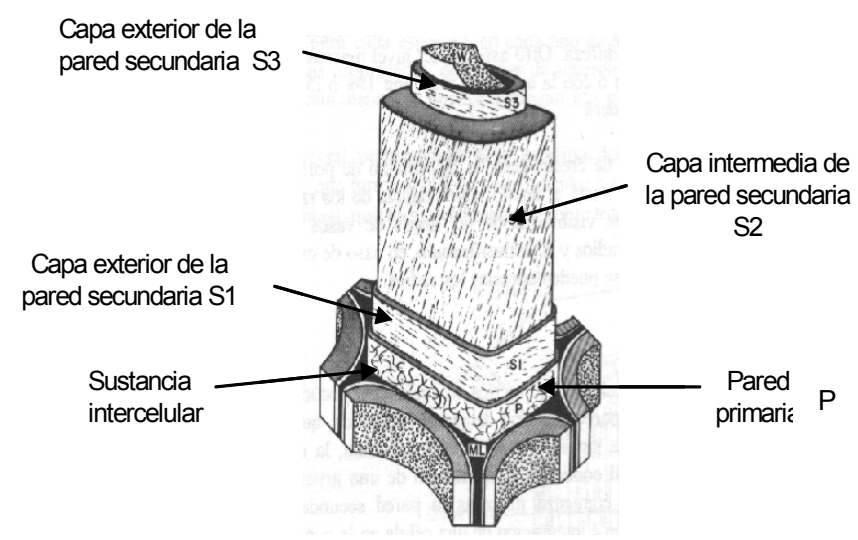

Fig. 1: Estructura simplificada de la pared celular de una fibra de celulosa.

Los autores antes mencionados postulan que la estructura de la pared de la fibra es del tipo multilaminar, esto se basa en los datos obtenidos por adsorción de nitrógeno y por observación en 
microscopia electrónica de la pared celular en la sección transversal. Se cree que las lámelas son concéntricas y su número depende del grado de hinchamiento de la pared celular. Cada una de las lámelas es menor a $100 \AA$ de espesor y su separación es de aproximadamente $35 \AA$. El área superficial específica de las fibras secas es de aproximadamente $1 \mathrm{~m}^{2} / \mathrm{g}$. Al utilizar la técnica de intercambio de solvente el área superficial específica puede llegar hasta $200 \mathrm{~m}^{2} / \mathrm{g}$. El tamaño de los poros en la pared celular varía entre 16 y $22 \AA$.

La naturaleza hidrofílica de las fibras celulósicas desempeña un papel importante, ya que el proceso de estructuración de la hoja de papel ocurre en un medio acuoso, por lo que las fibras absorben agua y se dispersan fácilmente James (1990). Cuando las fibras húmedas se unen durante el proceso de formación de la hoja, los enlaces se promueven por la atracción polar de las moléculas de agua con los grupos hidroxilo que cubren la superficie de la celulosa. Cuando se evapora el agua, los grupos hidroxilo de la superficie de la celulosa se unen por medio de puentes de hidrógeno.

La a-celulosa y hemicelulosas, tienen a lo largo de la macromolécula a intervalos regulares numerosos grupos ${ }^{-} \mathrm{OH}$ (hidroxilos) de gran afinidad con el agua, ésta se une a ellos por valencias polares y por puentes de hidrógeno. La asociación entre los grupos ${ }^{-} \mathrm{OH}$ y el agua es tan fuerte (adsorción) que aun cuando el papel esta seco contiene una proporción de agua que no es fácil de eliminar, de esta manera el agua contenida en las fibras del papel puede ser de tres clases: agua coloidal, agua capilar y agua de inhibición.

La capa externa de la pared celular de la fibra celulósica al contacto con agua inicia un proceso de hinchamiento gelatinoso, ya que la estructura se dilata en cuanto inicia la absorción de agua. Sólo las regiones microcristalinas entran en el proceso de hinchazón, los cristales de la a-celulosa no son penetrados por el agua y sólo su superficie puede participar en la absorción. Scallan (1977), utilizando la técnica de exclusión de soluto observa que el volumen acumulado de los poros (inaccesible para el agua) se incrementa sustancialmente en pulpas de abeto cuando estas se deslignifican hasta un rendimiento de $44 \%$. El hinchamiento de los derivados de la madera en medio líquido está en función de la fase líquida y de la composición química de las fibras. Atalla (1992), reporta que en las fibras recicladas aumenta la cristalinidad y disminuye el área superficial, a esto se debe la pérdida en la capacidad de hinchamiento de las fibras, lo que provoca una disminución de los enlaces fibra-fibra generando un papel más débil.

El mayor efecto en la fibra reciclada se observa después del primer ciclo, ya que en este punto es donde aparecen los cambios estructurales. Los cambios en cuanto a la cristalinidad de la estructura de la fibra con cada proceso de reciclado, Isogai y Atalla (1992) mencionan los siguientes aspectos:

La cristalinidad no se afecta por la cantidad de finos en la pulpa ni por la cantidad de hemicelulosas.

La cristalinidad no se modifica por la refinación.

Los cambios en la cristalinidad difieren de pulpa a pulpa.

El aumento en la cristalinidad puede originarse por el efecto del calor sobre la fibra durante la operación de secado.

Lindström y Carlsson (1982), mostraron que bajos niveles de sustitución de grupos hidroxilos son efectivos en disminuir el endurecimiento de la fibra y se necesita $1 \%$ de contenido de metoxilos para reemplazar el 2,2 \% de grupos hidroxilos. Concluyeron además que no todos los hidroxilos son sustituidos Fig. 2. Aparentemente, la ruptura de algunos enlaces del tipo puentes de hidrógeno es suficiente para disminuir el endurecimiento de las fibras.

Una de las alternativas que permite abrir los poros de la fibra para que el líquido penetre y la fibra se hinche es la adición de agentes de tensión superficial. En el marco del presente trabajo y para esta función se consideran los agentes de tensión superficial no iónicos: alcoholes grasos base etileno: alcohol tridietilico y alquil fenol base etileno: nonil fenol etoxilado.

El fenómeno de hornificación ha sido estudiado en fibra virgen, tal como lo reportan pública Mutjé et al. (2002) y García et al. (2000). Mientras que el presente trabajo pretende aportar bases en el conocimiento del fenómeno de hornificación en fibra reciclada. 


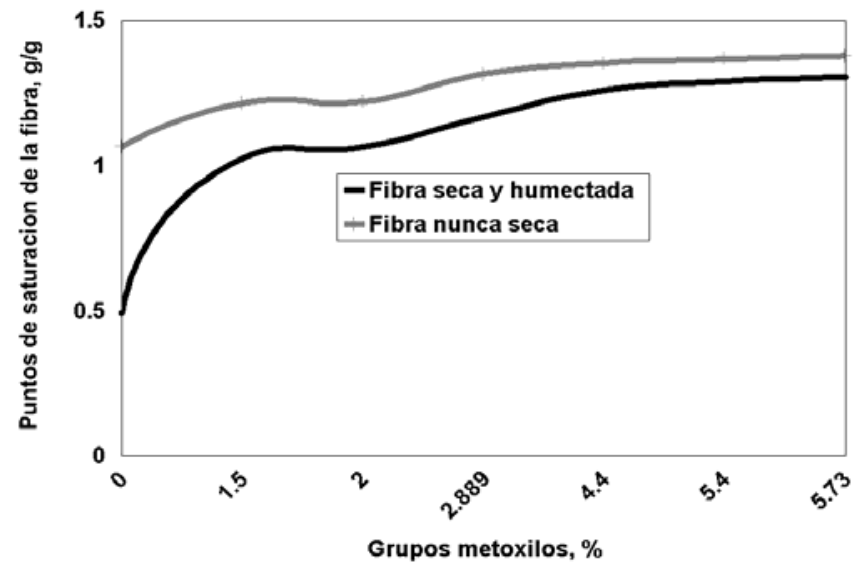

Fig. 2: Efecto de la metilación sobre el hinchamiento de pulpa kraft blanqueada

Los estudios para explicar el endurecimiento de la pared de las fibras celulosicas se han enfocado principalmente a pulpas de alto rendimiento: pulpas mecánicas, termomecánicas, químico-termomecánicas y en algunos casos pulpas químicas sin blanquear, pero sobre fibra reciclada hay poca información. En los casos estudiados el común denominador es la presencia de lignina en la pulpa, ésta tiene la capacidad de contraerse e hincharse en los procesos de secado y humedecimiento, lo que impide la formación de puentes de hidrógeno, evitando de esta forma el endurecimiento de las fibras y su total colapsamiento.

\section{MATERIALES Y METODOS}

En el marco de este trabajo se emplea fibra reciclada kraft de la primera generación denominada muestra A y fibra reciclada kraft de la generación " $n$ veces reciclada" denominada muestra B. Este estudio analiza el comportamiento de fibra reciclada frente a diferentes medios acuosos (humedecimiento) en virtud de la importancia que esta materia prima presenta en la fabricación de papel a nivel mundial, la metodología se desarrollan de acuerdo a la Fig, 3. Como variables de proceso se contemplan: medio acuoso, tiempo de contacto y como factores de respuesta se evalúan el Valor de Retención de Agua (WRV) y la Resistencia a la Tensión de los puntos óptimos determinados a partir de una mezcla de ambas fibras $50 \%$ A y $50 \%$ B. La hidratación se lleva a cabo en agua, y en medios acuosos modificados por la presencia de $\mathrm{NaOH}$, alcohol tridietilico y nonil fenol, estos productos se seleccionaron por su propiedad hidratante. El tiempo de contacto evaluado para cada medio hidratante fue de 0 a 24 h, generándose curvas de valores de hidratación para cada material.

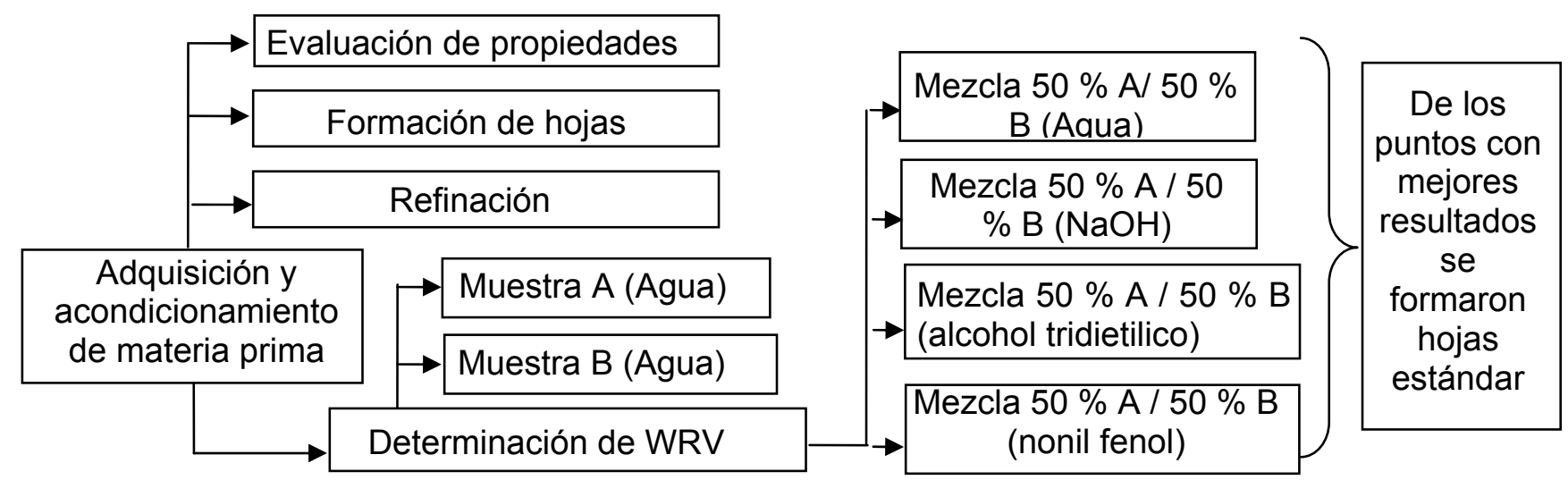

Fig. 3: Diagrama de desarrollo del trabajo

La caracterización de las materias primas y de los productos de la presente investigación se caracteriza de acuerdo a normas TAPPI (2007) (Technical Association of the Pulp and Paper Industry) y DIN (2007) (Deutsches Institut Für Normung e. V.) que se describen en la Tabla 1. 
Tabla 1, Normas internacionales.

\begin{tabular}{|l|l|}
\hline \multicolumn{2}{|c|}{ Normas empleadas en el estudio } \\
\hline Humedad: & TAPPI T-412 om-02 \\
\hline Ceniza: & TAPPI T-211 om-02 \\
\hline Clasificación de fibras, Bauer McNett: & TAPPI T-233 cm-95 \\
\hline Grado de refinación ${ }^{\circ}$ CSF: & TAPPI T-227 om-99 \\
\hline \multicolumn{2}{|c|}{ Evaluación de factores de respuesta } \\
\hline Formación de Hojas Estándar de Laboratorio & TAPPI T-205 sp-02 \\
\hline Tensión y (Elongación / Deformación) & TAPPI T- 494 om-01 \\
\hline Tensile Absorption Energy (T.E.A.) & TAPPI T- 494 om-01 \\
\hline Valor de Retención de Agua (WRV) & 417 Untersuchungs methoden-DIN \\
\hline
\end{tabular}

\section{RESULTADOS Y DISCUSIÓN}

Los resultados del análisis de las materias primas y el comportamiento de la absorción de agua por muestra se reportan el la Tabla 2.

Tabla 2: Caracterización de las muestras A y B.

\begin{tabular}{|c|c|c|}
\hline \multicolumn{1}{|c|}{ Determinación } & Muestra A & Muestra B \\
\hline $\begin{array}{l}\text { Valor de Retención de Agua } \\
\text { (WRV), 24 h }\end{array}$ & 1,10 & 1,39 \\
\hline Ceniza, \% & 2,22 & 0,86 \\
\hline${\text { Grado de Refinación, }{ }^{\circ} \mathrm{CSF}}^{\text {Retenido en mallas }}$ & 573 & 678 \\
\hline 30 & Retención: \% & Retención: \% \\
\hline 50 & 40,37 & 56,47 \\
\hline 100 & 10,75 & 6,47 \\
\hline 200 & 10,80 & 6,79 \\
\hline Finos & 14,47 & 14,68 \\
\hline
\end{tabular}

El análisis del comportamiento de cada materia prima fibrosa en función del tiempo de humectación en agua muestra que el valor inicial de WRV ( $\mathrm{g}$ agua $/ \mathrm{g}_{\text {fibra }}$ base seca) es de 0,34 para la muestra $\mathrm{A}$ y 0,4 para la muestra $\mathrm{B}$, al transcurrir $24 \mathrm{~h}$ de hidratación el valor alcanzado respectivamente es de 1,40 y 1,25. El comportamiento de ambas fibras en el proceso de hidratación durante el tiempo de evaluación registra en las Fig. 4 y 5 , para las muestras $A$ y $B$, en donde se observa que los valores de WRV son de un dígito, mientras que los valores reportados por otros autores para fibra virgen obtenida mediante proceso químico a partir de fibra de olivo y eucalipto son de tres dígitos. Mutjé et al. (2002), reportan valores de $128 \mathrm{WRV}$ ( $\mathrm{g}$ agua $/ \mathrm{g}_{\text {fibra }}$ base seca) para fibra sin refinar en ambas especies; y en fibra refinada de $50^{\circ} \mathrm{SR}\left(180^{\circ} \mathrm{CSF}\right)$ el WRV reportado es de 155 en fibra de olivo y 189 en fibra de eucalipto. Mientras que en fibra virgen de pulpa organosolv semiquímica de bagazo de caña (POB). García et al. (2000), reportan valores de 2 a 6 WRV ( $g_{\text {agua }} / g_{\text {fibra }}$ base seca).

El tiempo demandado por fibra celulosicas individuales para alcanzar su máximo valor de hidratación en agua, así como la exigencia de mezclar fibras celulosicas en el diseño de un nuevo papel para conservar y/o mejorar sus propiedades de uso conducen al análisis de mezclas fibrosas $50 \% \mathrm{~A}$ y 50 $\%$ B.

En la Fig 6. Se analiza el comportamiento de la mezcla de fibras $50 \%$ A / $50 \%$ B, en donde el máximo valor de absorción de agua es de 1,2 $\mathrm{g}_{\text {agua }} / \mathrm{g}_{\mathrm{fibra}}$, valor que se logra después de $18 \mathrm{~h}$ de contacto entre la fase acuosa y las fibras. 


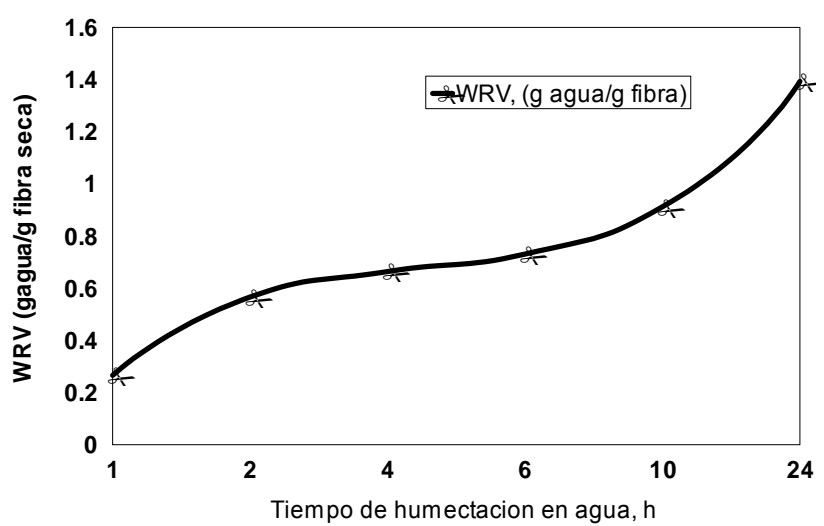

Fig. 4: WRV de muestra A en función del tiempo de humectación con Agua.

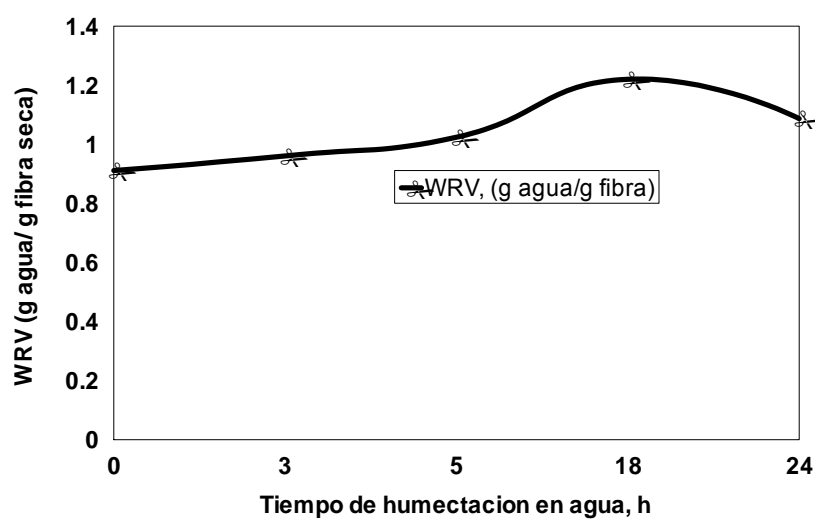

Fig. 6: WRV de mezcla de fibras A/B en función del tiempo de humectación (Agua).

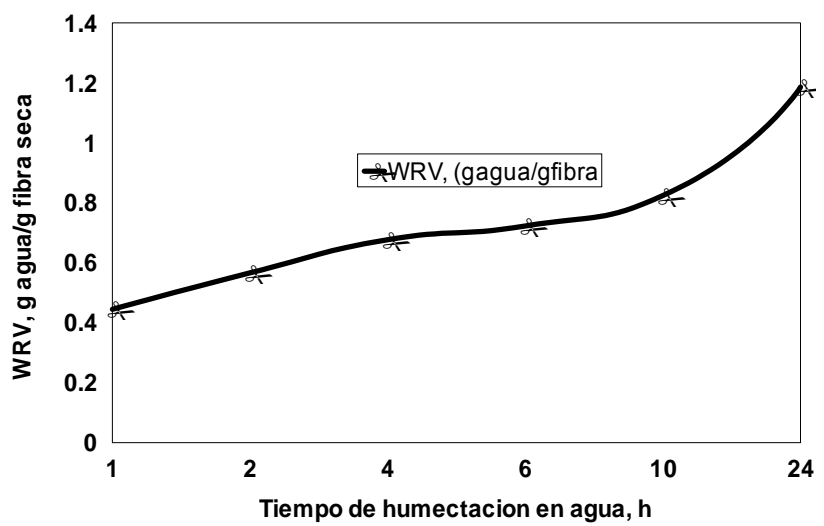

Fig. 5: WRV de muestra B en función del tiempo de humectación con Agua

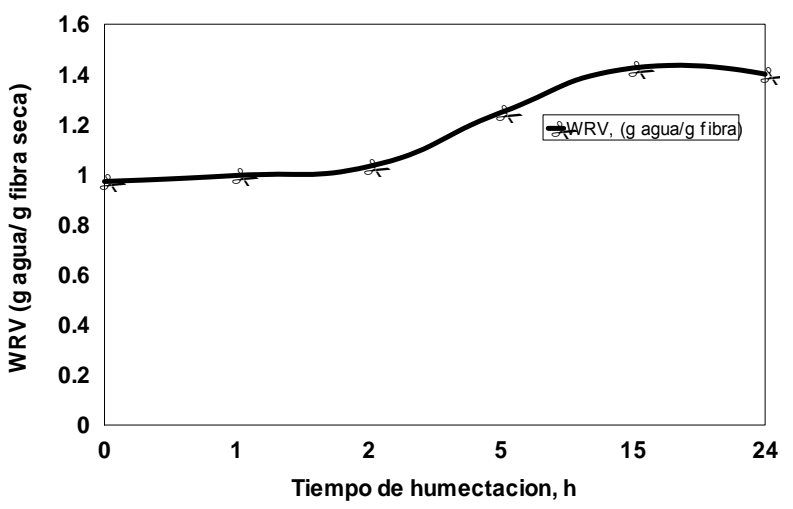

Fig. 7: WRV de mezcla de fibras A/B en función del tiempo de humectación $(\mathrm{NaOH})$

Al evaluar el comportamiento de la mezcla de fibras en medio alcalino con sosa $(\mathrm{NaOH}, \mathrm{pH}=11)$, el máximo valor de absorción de agua $1,4 \mathrm{~g}$ agua $/ \mathrm{g}_{\text {fibra }}$ se registra a las $12 \mathrm{~h}$ (Fig. 7), el incremento de tiempo para llegar a este valor entre $\mathrm{NaOH}$ y agua es de $6 \mathrm{~h}$ y el valor se incrementa en 0,2 $\mathrm{g}_{\mathrm{agua}} / \mathrm{g}_{\text {fibra. }}$. De las Fig. 4 y 5 se observa que el valor del WRV de fibra reciclada es bajo con relación al valor WRV de pulpa semiquímica de bagazo de caña y mucho más bajo si se comparan estos valores con los valores reportados para fibra virgen de un proceso químico a partir de otras especies.

En la Fig 8, se muestra el impacto sobre el valor de WRV de la mezcla fibrosa al emplear como agente humectante alcohol tridietilico, AT al 0,05 \% en base a fibra seca (b.s.), siendo el valor WRV $1,2 \mathrm{~g}$ agua $/ \mathrm{g}_{\mathrm{fibra}}$, el cual se alcanza en $1 \mathrm{~h}$.

En la Fig 9, se muestra el impacto sobre el valor WRV de la mezcla fibrosa al emplear como agente humectante nonil fenol al $0,05 \%$ en base a fibra seca (b.s.), siendo el valor WRV 1,8 $\mathrm{g}$ agua $/ \mathrm{g}_{\mathrm{fibra}}$, el cual se alcanza en $5 \mathrm{~h}$ de contacto.

El empleo de agentes de tensión superficial (ATS) parece ser muy ventajoso en el proceso de hidratación de las fibras, tal como se desprende de las Fig. 8 y Fig. 9, ya que el máximo valor de WRV se alcanza en menor tiempo en comparación con agua y en medio acuoso con $\mathrm{NaOH}$. Sin embargo, al analizar los resultados de la Fig. 10 se observa que la menor resistencia a la tensión $(2,5$ $\mathrm{kg}_{\mathrm{f}} / 15 \mathrm{~mm}$ ) se alcanza cuando la mezcla fibrosa se humecta solo en agua y el mayor valor de resistencia a la tensión $\left(4,4 \mathrm{Kg}_{\mathrm{f}} / 15 \mathrm{~mm}\right)$ se logra cuando se emplea $\mathrm{NaOH}$ en el medio acuoso. En el desarrollo de las propiedades de resistencia a la tensión $\left(\mathrm{Kg}_{\mathrm{f}} / 15 \mathrm{~mm}\right)$, elongación $(\%)$ y TEA $\left(\mathrm{J} / \mathrm{m}^{2}\right)$ los agentes de tensión superficial no mostraron una ventaja importante en comparación a los 
resultados generados al aplicar de $\mathrm{NaOH}$ en el medio acuoso. La máxima elongación se logro cuando la mezcla fibrosa se trata con $0,05 \%$ de AT $(2,2 \%)$ mientras que al tratar la mezcla fibrosa con $\mathrm{NaOH}$ se alcanzo un valor de $1,8 \%$. El máximo valor de TEA $\left(43,5 \mathrm{~J} / \mathrm{m}^{2}\right)$ se alcanza al tratar la mezcla fibrosa con $\mathrm{NaOH}$, mientras que al tratar la mezcla fibrosa con 0,05 de NF se alcanza un valor de $32,3 \mathrm{~J} / \mathrm{m}^{2}$, siendo este el valor más próximo al reportado en medio alcalino.

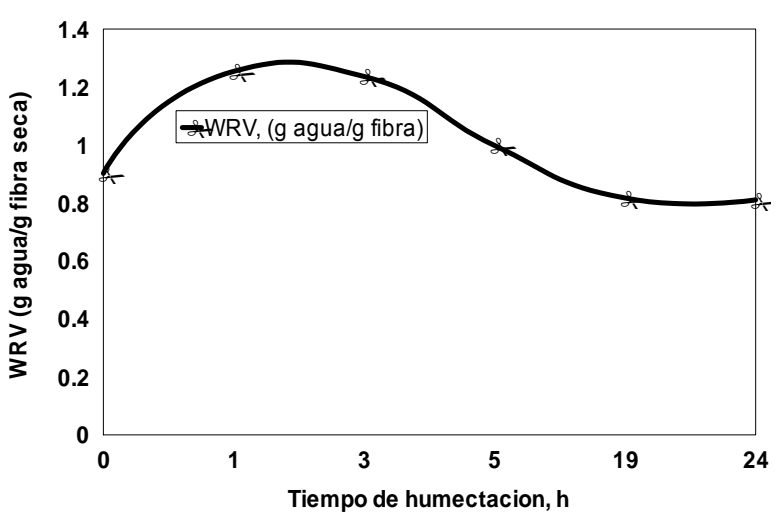

Fig. 8: WRV de mezcla de fibras A/B en función del tiempo de humectación (AT)

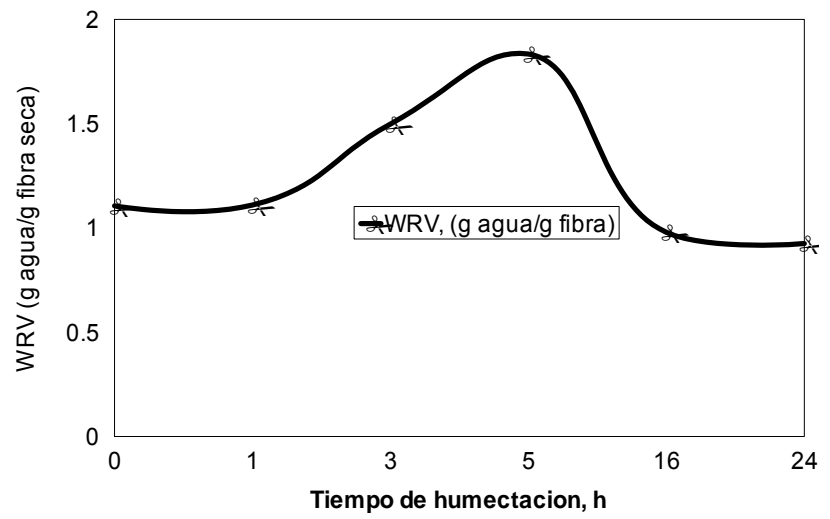

Fig. 9: WRV de mezcla de fibras A/B en función del tiempo de humectación (NF)

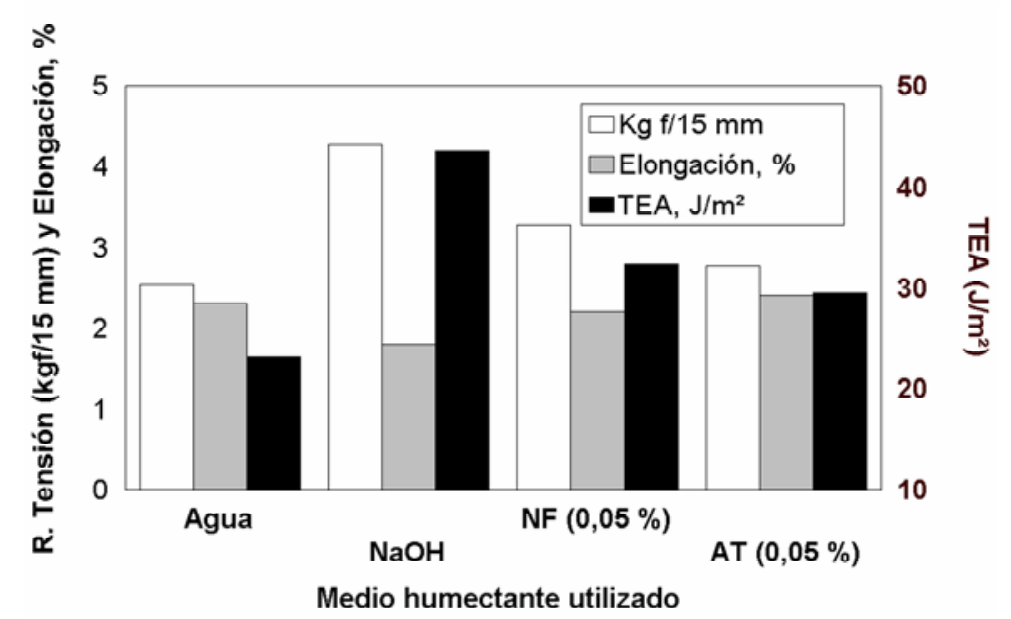

Fig. 10: Resistencia a la tensión, elongación y TEA

\section{CONCLUSIONES}

La fibra celulosica con un proceso de reciclado alcanza en $24 \mathrm{~h}$ un valor de WRV de $1,4 \mathrm{~g}_{\text {agua }} / \mathrm{g}_{\text {fibra }}$ y la fibra celulosica con $\mathrm{n}$ ciclos de reciclado alcanzo un valor de WRV de 1,2 $\mathrm{g}_{\text {agua }} / \mathrm{g}_{\mathrm{fibra}}$ en $24 \mathrm{~h}$ de hidratación en agua.

Los valores de WRV en mezcla fibrosa $50 \%$ A / $50 \%$ B en agua registran valores de $1,2 \mathrm{~g}_{\text {agua }} / \mathrm{g}_{\text {fibra }}$ en $18 \mathrm{~h}$, al aplicar medio acuoso con presencia de $\mathrm{NaOH}$ el valor de WRV es de $1,4 \mathrm{~g}$ agua $/ \mathrm{g}_{\text {fibra }}$ en 15 h., mientras que al utilizar nonil fenol al $0,05 \%$ el valor de WRV alcanzado es de $1,8 \mathrm{~g}_{\text {agua }} / \mathrm{g}_{\text {fibra }}$ en 5 h., y al utilizar alcohol tridietilico, AT al $0,05 \%$ el valor de WRV alcanzado es de $1,3 \mathrm{~g}$ agua $/ \mathrm{g}_{\text {fibra }}$ en $1 \mathrm{~h}$.

El valor de retención de agua de la mezcla fibrosa mejoran del orden de $15 \%$ con la adición de agentes de tensión superficial, específicamente alcohol tridietilico al $0,05 \%\left(1,4 \mathrm{~g}_{\text {agua }} / \mathrm{g}_{\mathrm{fibra}}\right)$ en comparación del medio alcalino con $\mathrm{NaOH}\left(1,2 \mathrm{~g}_{\text {agua }} / \mathrm{g}_{\text {de fibra }}\right)$.

La resistencia a la tensión y el TEA, alcanzan valores más altos de 4,3 y 43,5 kgf/15mm respectivamente cuando se emplea $\mathrm{NaOH}$, mientras que la elongación máxima se alcanza al aplicar alcohol tridietilico al $0,1 \%$ es de $2,4 \%$. 


\section{REFERENCIAS}

Atalla, Rajai H.; "Structural changes in cellulose during papermaking and recycling", Material Research Society. Symp. Proc.: 266, 229-236 (1992).

DIN - ISO 23714; Faserstoffe- Bestimmung des Waserrrückhaltewertes (WRV), Deutsches Institut für Normung, e. 08-31-2007.

Fengel, D. y G. Wegener; "Wood - Chemistry, ultrastructure - reactions", ISBN 311008481 3, 1, 7678 Editorial Walter de Gruyters. New York (1984)

García, A. y otros seis autores; "Potencialidades de la refinación de pulpa organosolv de bagazo en la producción de papel corrugado en Cuba”. CIADICYP 2000, Iguazu, Argentina (2000)

Isogai, A. y R.H. Atalla; "Preparation of cellulose-chitosan polymer blends", Carbohydrate Polymer: 19, 25-28 (1992)

James P. Casey; "Pulpa y Papel. Química y tecnología química"; Editorial LIMUSA, ISBN 96818 2061 429-64, México (1990);

Jayme G.; Mikro-Quellungsmessungen an Zellstoffen. Wochenbl. Papierfabr: 6, 187-194 (1994)

Lindström, T. y G. Carlsson; "The Effect of Carboxyl Groups and their lonic Form during Drying on the Hornification of Cellulose Fibers", Svensk Papperstidning: 85(15), 146 -151 (1982).

Minor, J.L.; "Strength loss in recycled fibers and methods of restoration" Material Research Society Symp.: 266, 215-228 (1992),

Mutjé P. y otros cuatro autores; "Estudio comparativo de la demanda catiónica de las suspensiones de pastas crudas de poda de olivo y eucalipto", CIADICYP- Brasil (2002),

Scallan A.M.; "The accommodation of water within pulp fibers. Fiber Water Interactions in PaperMaking", Oxford Technical Division, The British Paper Board Industry Federation: 19-23 (1977).

Stone, J.E. y A.M. Scallan; "A study of cell wall Structure by Nitrogen Adsorption". Pulp paper Magazine of Canada: 8, 407-714 (1965).

TAPPI; Official Test Methods, Technical Association of the Pulp and Paper Industry (2007) 